\title{
Reducing the measurement uncertainty of shutter-less microbolometer-based infrared measurement systems
}

\author{
A. Tempelhahn, H. Budzier, V. Krause, G. Gerlach \\ Solid-State Electronics Laboratory, Technische Universität Dresden, 01062 Dresden, Germany, \\ alexander.tempelhahn@tu-dresden.de
}

\begin{abstract}
Shutterless infrared cameras based on microbolometer focal plane arrays (FPAs) are the most widely used cameras in thermography, in particular in the fields of handheld devices and small distributed sensors. For acceptable measurement uncertainty values the disturbing influences of changing thermal ambient conditions have to be treated corresponding to temperature measurements of the thermal conditions inside the camera. We propose a compensation approach based on calibration measurements where changing external conditions are simulated and all correction parameters are determined. This allows to process the raw infrared data and to consider all disturbing influences. The effects on the pixel responsivity and offset voltage are considered separately. The responsivity correction requires two different, alternating radiation sources. This paper presents the details of the compensation procedure and discusses relevant aspects to gain low temperature measurement uncertainty.
\end{abstract}

Key words: microbolometer FPA, thermal drift compensation, shutter-less, TEC-less

\section{Introduction}

Microbolometer-based infrared cameras are used in many fields of application, e.g. in quality control during production processes, fire protection, and surveillance. Their benefits are low power consumption due to uncooled thermal infrared sensors, compact size and low costs compared to infrared cameras based on cooled photon detectors. The latest improvements in the microfabrication process of microbolometer focal plane arrays (FPAs) in terms of pixel pitch leads to increasing spatial resolution but reduced sensor dimensions. The decreasing sensor cost is another reason why infrared thermography enters new fields of application, e.g. smart phone devices or sensors for smart building control systems. But radiometrically calibrated infrared cameras use commonly optical shutters for runtime recalibration purposes in order to overcome thermal drift influences on the measurement. This mechanically moved part has to cover the entire aperture, and hence, represents one of the size-limiting components of the infrared camera. For that reason, shutter-less infrared cameras are required for these new fields of application. This paper presents the procedure to compensate the disturbing influences of a changing ambient temperature and discusses relevant aspects to gain low temperature measurement uncertainty.

\section{Thermal Drift}

The signal voltage $V_{p i x, i j}$ of a pixel $(i j)$ is linearly related to the exchanged radiant flux $\Phi_{p i x, i j}$ of this pixel [1]. This relation comprises the pixel voltage responsivity $R_{V, i j}$ and the offset voltage $V_{0, i j}$ :

$$
V_{p i x, i j}=R_{V, i j} \Phi_{p i x, i j}+V_{0, i j} \text {. }
$$

Pixel responsivity and offset voltage vary over the sensor array due to little variations during the microfabrication process of the sensor array. Additionally, the values of both sensor parameters change according to the drifting sensor temperature. The exchanged radiant flux $\Phi_{p i x, i j}$ depends on the camera design, especially the $f$-number of the used aperture defining the projected solid angle $\omega_{f o v}$ related to the camera field of view (FOV). State-of-the-art infrared cameras use apertures with an $f$-number around unity. In [2] we studied the pixel field of view respectively its projected solid angle and demonstrated that it covers nearly the entire half space $\omega_{h s}$. The pixel location and its distance to the optical axis results in a pixel-specific projected solid angle $\omega_{f o v, i j}$. The entire pixel projected solid angle $\omega_{\text {pix }}$ is composed of parts $\omega_{f o v, i j}$ related to the 
scene radiation and $\omega_{c a m, i j}$ related to the radiation derived from the camera interior:

$$
\omega_{p i x}=\omega_{f o v, i j}+\omega_{c a m, i j} \approx \omega_{h s} .
$$

Changing ambient conditions, especially the ambient temperature, affect the thermal conditions inside the camera and the sensor temperature due to heat conduction and convection. These influences have huge effect on the absolute temperature measurement uncertainty and the spatial deviation of the pixel values if the camera looks at a homogeneous radiation source.

We propose a calibration method which enables correcting the temperature-dependent sensor parameters based on runtime measurements of the sensor temperature and an estimation of the disturbing camera radiation using additional temperature probes placed inside the camera housing. This method combines experiences of our previous works on calibration of infrared cameras with shutters [3] and shutter-less infrared cameras using temperature-stabilized microbolometer FPAs [4].

\section{Infrared Camera}

The compensation procedure will be explained for an infrared camera based on a ULIS microbolometer sensor array without temperature stabilization (Tab. 1) [5].

Tab. 1: Properties of the used infrared camera.

\begin{tabular}{|l|l|}
\hline Sensor type & $\begin{array}{l}\text { UL03162-028 (ULIS, } \\
\text { France) }\end{array}$ \\
\hline TEC & w/o \\
\hline NETD & $\begin{array}{l}<100 \mathrm{mK}(\mathrm{F} / 1,300 \mathrm{~K}, \\
50 \mathrm{~Hz})\end{array}$ \\
\hline Resolution & $384 \times 288$ \\
\hline Pixel pitch & $25 \mu \mathrm{m}$ \\
\hline Uniformity (deviation) & $<1.5 \%$ \\
\hline Power consumption & $<100 \mathrm{~mW}$ \\
\hline f-number & 1.0 \\
\hline Focal length & $18 \mathrm{~mm}$ \\
\hline
\end{tabular}

Three temperature probes (LM61, Texas Instruments, USA) are placed inside the camera housing in order to capture camera temperature changes. The estimation of the disturbing camera radiation is based on these temperature values. Figure 1 depicts the positions of the three temperature probes (TP). These four temperatures, three camera and the sensor temperature, form the correction input and are continuously captured during the measurements.

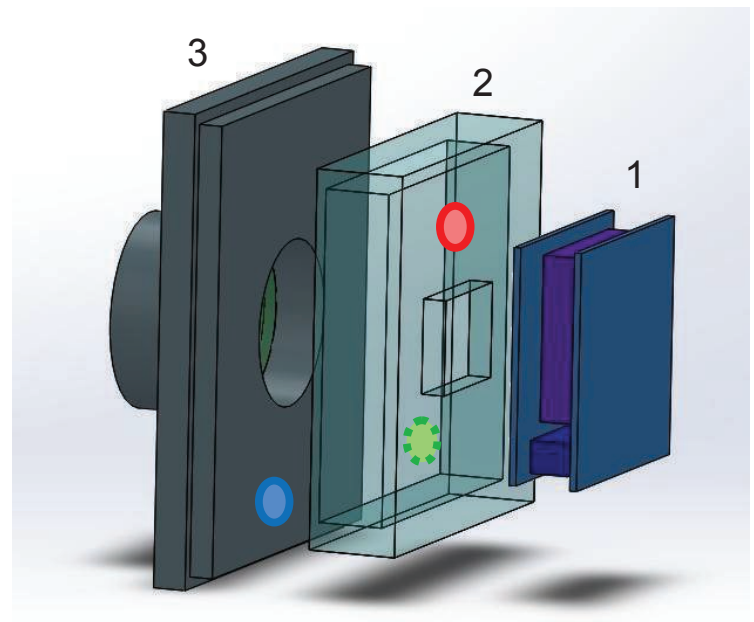

Fig. 1: Positions of the three temperature probes inside the camera housing. TP\#1 (red) is placed close to the signal processing unit with the sensor array (1) on the back side of the optical channel (2). TP\#2 (dashed green) is located on the front side of the optical channel (2). TP\#3 is placed on the front panel (3) carrying the optics.

\section{Calibration}

During real measurements the ambient temperature might change over time. We assume that the current ambient temperature is uniformly distributed around the camera housing and that its changes are uniform as well. During the calibration these changing external conditions are simulated using a heating chamber which allows controlling the ambient temperature by defining a temperature time regime. The infrared camera is placed inside this heating chamber (Fig. 2). The heating chamber has a sidewise opening which allows the camera to look at different blackbodies or testing scenes.

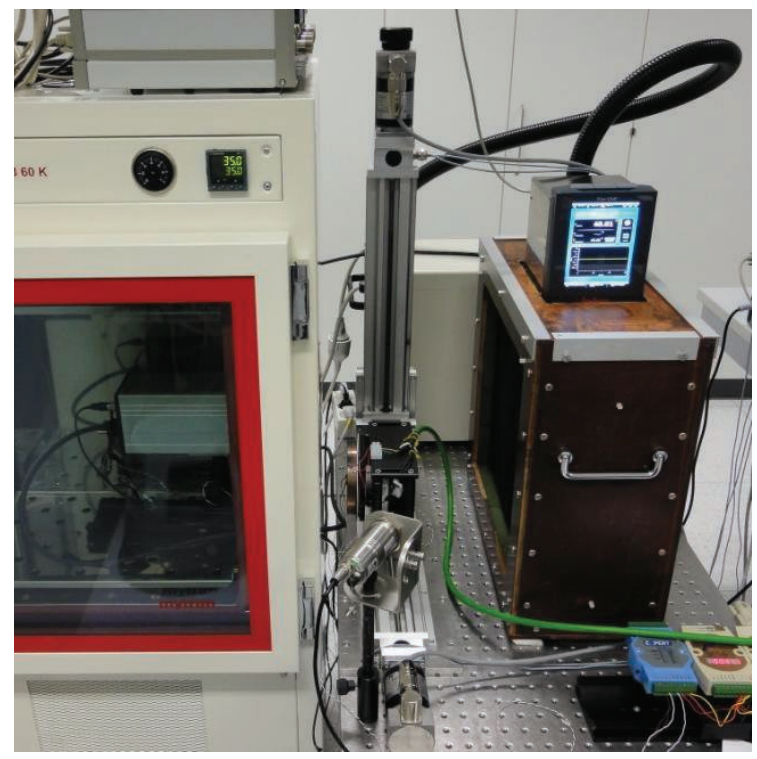

Fig. 2: Calibration set-up comprising heating chamber (left) with the infrared camera inside and a 
sidewise opening, and one large panel blackbody (right) positioned in front of the opening. A second small round blackbody (middle) can be moved in front of the camera.

The temperature compensation approach consists of three steps: (i) non-uniformity correction (NUC), (ii) correction of the sensor temperature-dependent responsivity changes and (iii) offset correction based on the sensor and the camera temperatures. The corrected pixel signal voltages are converted into temperature values according to a radiometric calibration transfer function [3] afterwards. Each calibration step is followed by bad pixel replacement procedure.

The raw pixel signal voltage $V_{\text {raw }, i j}$ comprises the exchanged radiant flux density $E_{\text {pix }, i j}$ multiplied by the pixel responsivity $R_{V, i j}$ and the pixel area $A_{\text {pix }}$, and the pixel offset voltage $V_{0, i j}$ :

$$
V_{r a w, i j}=R_{V, i j}\left(\vartheta_{f p a}\right) A_{p i x} E_{p i x, i j}+V_{0, i j}\left(\vartheta_{f p a}\right) \text {. }
$$

The correction of the pixel responsivity $R_{V, i j}$ is based on the measurements of two different radiation sources with known constant temperature difference under changing ambient conditions. Two different panel blackbodies were used: (i) a rectangle water bath blackbody with a radiant surface of $350 \mathrm{~mm} \times 350 \mathrm{~mm}$ and (ii) a round copper plate blackbody with a coated radiant surface of $120 \mathrm{~mm}$ diameter that can be moved in-between the infrared camera and the other blackbody. Both blackbodies cover the entire field of view of the camera. The delay between two consecutive measurements amounts to $45 \mathrm{~s}$.

Beside the responsivity, also the offset voltage $V_{0, i j}$, the pixel radiant exitance $M_{\text {pix }}$ and the camera irradiance $E_{c a m, i j}$ influence the raw signal voltage $V_{\text {raw } i j}$. The exchanged radiant flux density $E_{p i x, i j}$ during the offset calibration is composed of the object irradiance $E_{o b j, i j}$ derived from a constant and uniform radiating surface, the camera irradiance $E_{c a m, i j}$ minus the pixel radiant exitance $M_{\text {pix }}$ :

$$
E_{p i x, i j}=E_{o b j, i j}+E_{c a m, i j}\left(\vartheta_{c a m}\right)-M_{p i x}\left(\vartheta_{f p a}\right) .
$$

The offset voltage $V_{0, i j}$ and the pixel radiant exitance $M_{\text {pix }}$ are related to the sensor temperature $\vartheta_{f p a}$. The camera irradiance $E_{c a m, i j}$ depends on the temperature distribution inside the camera measured by the temperature probes.

The calibration procedure takes much time because of the large time constant of the infrared camera housing. The calibration set-up of Fig. 2 enables to determine the correction coefficients in a single temperature-time regime run. All four temperatures are captured during this procedure as well as the temperatures of the two blackbodies. Figure 3 shows the temperature responses of the temperature probes TP\#1...3, the sensor temperature $\vartheta_{f p a}$ and the temperatures $\vartheta_{\text {water }}$ and $\vartheta_{\text {copper }}$ of the two blackbodies versus time. When the thermal conditions have settled then the raw infrared images are used for NUC.

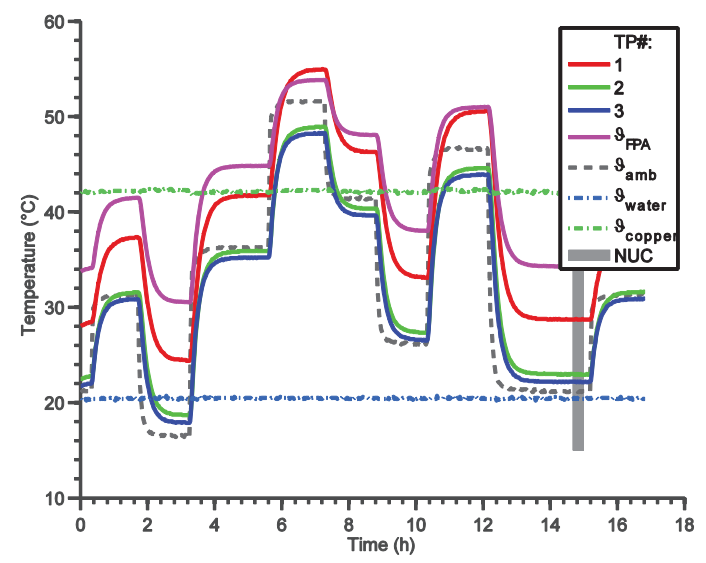

Fig. 3. Temperature responses of the temperature probes TP\#1...3, the sensor temperature $\vartheta_{f p a}$ and the temperatures $\vartheta_{\text {water }}$ and $\vartheta_{\text {copper }}$ of the two blackbodies versus time during calibration regime. The ambient temperature $\vartheta_{a m b}$ is controlled between $15{ }^{\circ} \mathrm{C}$ and $50{ }^{\circ} \mathrm{C}$. The temperature probes and the sensor temperature follow the changes. Both blackbody temperatures are constant. Grey bar marks steady-state conditions for NUC.

\section{Non-uniformity correction}

The NUC equalizes small variations of the pixel parameters (responsivity and offset voltage) and the different pixel responses due to the pixel-dependent projected solid angle $\omega_{f o v, i j}$ [3]. The input data are at least two raw infrared images of a panel blackbody covering the entire camera FOV at the thermal steady state. A standard two-point-NUC is sufficient because of the linear relation between radiation and signal voltage (see Eq. (1)). The correction using the two coefficient matrices gain $_{i j}$ and of $f_{i j}$ yields:

$$
V_{n u c, i j}=\operatorname{gain}_{i j} V_{\text {raw }, i j}+o f f_{i j} .
$$

After this correction all pixel follow the same response curve. However, this uniform response is related to specific thermal conditions $\left(\vartheta_{a m b}, \vartheta_{c a m}, \vartheta_{f p a}\right)$ outside and inside the camera. This captured temperature values form a set of reference temperatures. Changes of these thermal conditions and their effects on the temperature measurement are treated in the next correction steps. 


\section{Responsivity correction}

It is assumed that all pixels of the sensor array have the same temperature. An all-time uniform sensor temperature results in equal responsivity changes for all pixels. But it is not possible to distinguish parts of the signal voltages related to different portions of radiation according to their origin, or to illuminate all pixels with the same radiant flux density. For that reason and due to pixel-dependent projected solid angle $\omega_{f o v, i j}$ the responsivity changes are pixel-dependent. The difference signal voltage $\Delta V_{o b j, i j}$ depends on the pixel responsivity $R_{V, i j}$, the pixel area $A_{\text {pix }}$, the projected solid angle $\omega_{f o v, i j}$ and the radiant exitance difference $\Delta L_{o b j}$ :

$$
\Delta V_{o b j, i j}\left(\vartheta_{f p a}\right)=R_{V, i j}\left(\vartheta_{f p a}\right) \cdot A_{p i x} \omega_{f o v, i j} \Delta L_{o b j} .
$$

During the calibration both blackbodies are switched periodically. The time deviation between two consecutive measurements is considered by time-based averaging in order to get quasi-simultaneous measurements. Figure 4 shows the measured difference signal voltage $\Delta V_{o b j, i j}$ versus the sensor temperature $\vartheta_{f p a}$ for three sample pixels with different distances from the optical axis. The curves have different slopes and cross each other at the reference sensor temperature due to the previously applied NUC. A polynomial of the second order is sufficient for regression:

$$
g_{V, i j}\left(\vartheta_{f p a}\right)=1+g_{1, i j} \vartheta_{f p a}+g_{2, i j} \vartheta_{f p a}^{2} .
$$

The normalized regression function $g_{V, i j}$ is used to correct the NUC signal voltage $V_{n u c, i j}$ :

$$
V_{G, i j}=\frac{V_{n u c, i j}}{g_{V, i j}\left(\vartheta_{f p a}\right)} \text {. }
$$

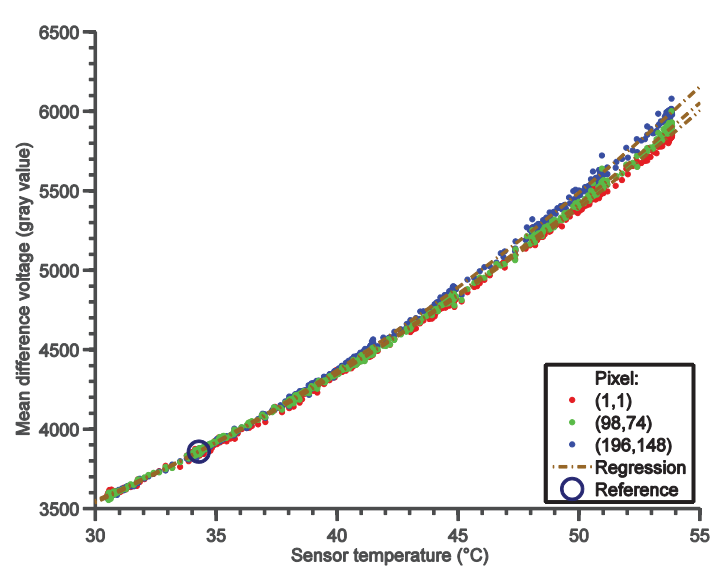

Fig. 4. Difference signal voltage versus sensor temperature of three sample pixels at the corner of the FPA (red), in the middle (blue) and in-between (green). The circle marks the reference sensor temperature from the NUC where the different curves cross each other.

\section{Offset voltage correction}

In [4] possibilities have been studied to compensate changing disturbing camera radiation using temperature-stabilized sensor arrays. An FPA without temperature stabilization makes the offset correction more complex since the pixel offset voltage $V_{0, i j}$ and the radiant exitance $M_{\text {pix }}$ depend on the sensor temperature (see Eqs. (3), (4)). It turned out that additional temperature probes provide information which help reducing the measurement uncertainty, especially in cases of abrupt ambient temperature changes. An offset correction approach for TEC-less microbolometers is presented in [6] using the sensor temperature and its time derivatives for correction.

Figure 5 shows the signal voltage after responsivity correction versus all accessible temperatures. The steady-state regression shows that second-order polynomials are sufficient for the relation between the signal voltage and the camera temperature probes. A polynomial of the third order is needed for the regression based on the sensor temperature and agrees with the manufactures specification.

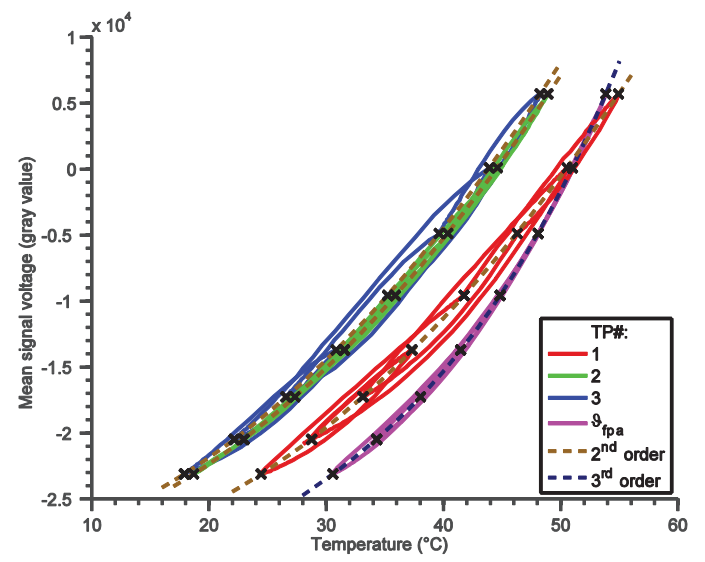

Fig. 5. Relation between mean signal voltage and different temperature compensation inputs (TP\#1...3, sensor temperature) during calibration regime. Black crosses mark the steady-state conditions used for the regression (dashed lines).

Using only one steady-state regression is insufficient for transient thermal conditions. Additional information about the temperature distribution inside the camera is provided by the time derivatives of the camera temperatures. Even if the signal voltage is only affected by the absolute sensor temperature (see Eqs. (3), (4)) the time derivatives of the sensor temperature do increase the regression accuracy. Figure 6 shows the relation between the steady-state 
corrected signal voltage and the first time derivatives of the four temperatures.

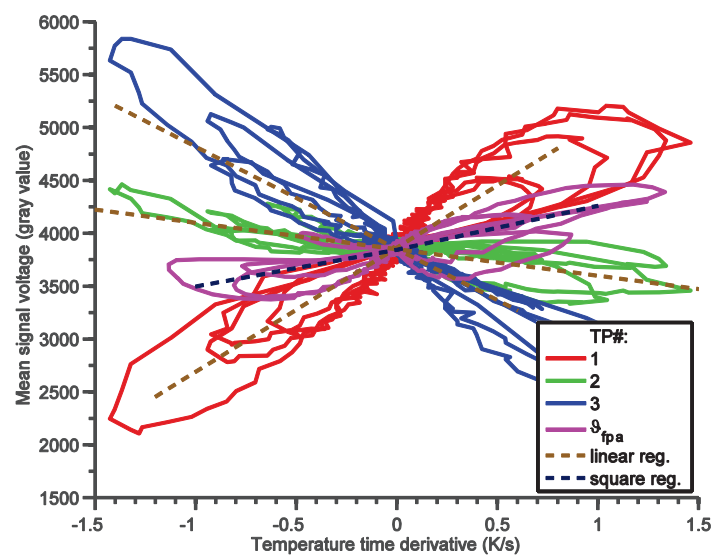

Fig. 6. Relation between the mean steady-state corrected signal voltage and the first time derivatives of TP\#1...3 and the sensor temperature (straight lines), and resulting regression functions (dashed lines).

Another possibility to increase the regression accuracy is to take cross-correlation coefficients into account which are formed by multiplying two or more camera temperature inputs [4].

The final regression model is composed of the following three groups of inputs: (i) absolute temperatures, (ii) time derivatives and (iii) cross-correlation of temperature inputs. The resulting correction based on the regression function $o_{V, i j}$ yields:

$$
V_{O, i j}=V_{G, i j}-o_{V, i j}\left(\vartheta_{f p a}, \vartheta_{T P m}, \vartheta_{T P m} \cdot \vartheta_{T P n}, \ldots\right) .
$$

Table 2 compares the correction results of different compositions of temperature inputs. The time derivative inputs are more important than the cross-correlation. It can be seen that the more coefficients are used the better are the correction results.

\section{Verification measurement}

The compensation functions were evaluated in a second time series measurement based on a testing scene comprising four small individual blackbodies. This test scene allows the observation of four different temperature-controlled surfaces at the same time. A fifth region of interest (ROI) is defined on the coated border frame. Figure 7 shows the corrected infrared image and the location of the five ROls. The temperature-time regime was strongly reduced compared to the calibration procedure. Thus, the camera does not reach the thermal steady state. This shows the limitations of the correction approach considered here. Figure 8 depicts the captured temperature responses during that verification measurement.

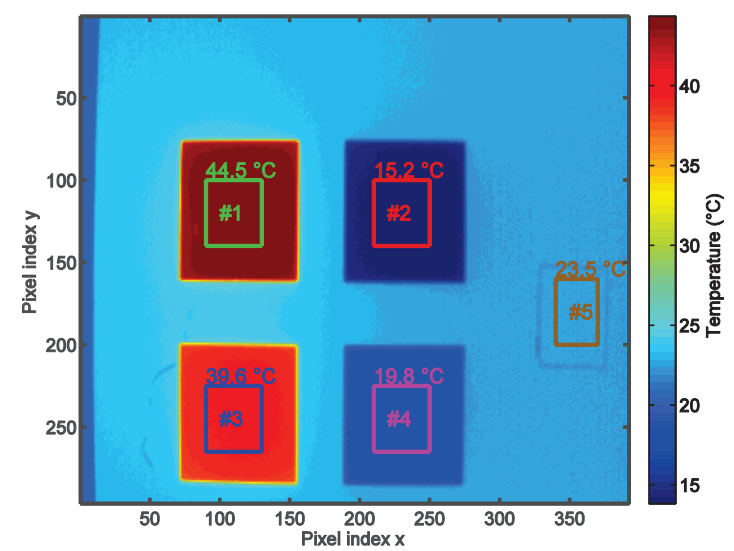

Fig. 7. Infrared image of the testing scene after correction using the best combination of the temperature inputs (see Tab. 2) and after applied conversion from voltage into temperature. Squares mark the five evaluated ROIs with enumeration and absolute temperatures measured with a reference pyrometer.

The corrected mean voltage and the spatial standard deviation of the five ROls are evaluated and added to Table 2 . The achieved measurement uncertainty of the second measurement was significant higher. This might be due to the non-ergodic behavior of heat conduction inside the camera under switching transient thermal conditions [3]. The offset function with the best correction results has changed compared to the calibration results. One reason might be the relatively large time period of $90 \mathrm{~s}$ between two consecutive measurements during the offset calibration. Nevertheless, the best offset correction function achieves temporal mean temperature deviations of $(208 \ldots 417) \mathrm{mK}$ and mean spatial deviations of $(60 \ldots 93) \mathrm{mK}$ for the five ROls over the entire measurement time. During steady-state conditions these values change to (88...100) $\mathrm{mK}$ and $(57 . .106) \mathrm{mK}$ respectively.

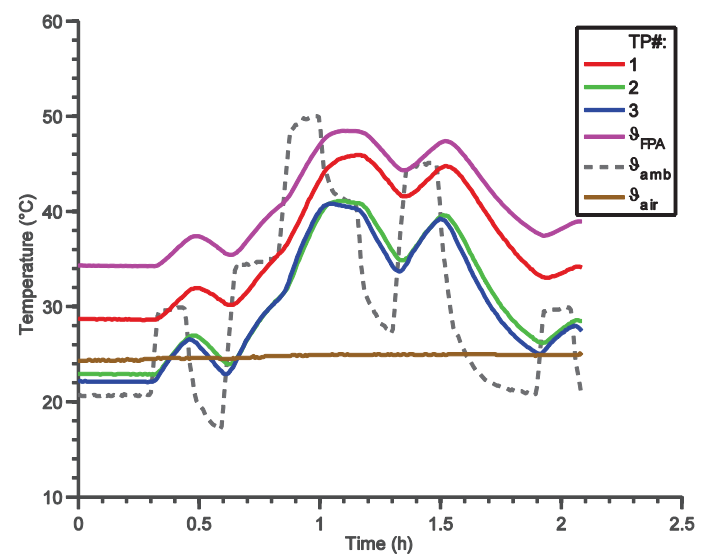

Fig. 8. Responses of the correction inputs TP\#1...3 and the sensor temperature versus time during verification measurement. At the beginning the camera is kept under steady-state conditions. After $20 \mathrm{~min}$ the ambient temperature is controlled 
between $15^{\circ} \mathrm{C}$ and $50{ }^{\circ} \mathrm{C}$ but with decreased hold time compared to the calibration regime.

\section{Conclusion}

In this paper we presented an ambient temperature compensation method for microbolometer-based infrared cameras working without shutter and sensor temperature stabilization. The responsivity and offset correction were based on several temperature inputs representing the thermal state inside the camera. The measurement uncertainty after correction depends primarily on the numbers of considered coefficients for the offset correction function. A temporal mean temperature deviation of $79.8 \mathrm{mK}$ and a mean spatial deviation of $40.3 \mathrm{mK}$ were achieved for the calibration measurement. The deviation results of a verification measurement were significant higher. The sources for the differences in correction uncertainty will be considered in the future work. The separate calibration of responsivity and offset in two time series measurements might help to decrease the residual measurement uncertainty. This would allow reducing the measurement period for the offset calibration.

Tab. 2: Comparison of offset correction results using different numbers of temperature inputs. Bold numbers show the best input selection for the calibration time series measurement and for the verification measurement.

\begin{tabular}{|l|l|l|l|l|l|}
\hline Temperature input & \multicolumn{2}{|l|}{$\begin{array}{l}\text { Residual temporal } \\
\text { standard deviation } \sigma_{t} \text { of } \\
\text { the mean corrected pixel } \\
\text { signal voltages (gray } \\
\text { value) } \\
\text { Calibration } \\
\text { (full array) }\end{array}$} & $\begin{array}{l}\text { Vesidual mean spatial } \\
\text { standard deviation } \sigma_{s} \text { of } \\
\text { (ROI areas) }\end{array}$ & $\begin{array}{l}\text { (Rorrected pixel } \\
\text { voltages (gray value) }\end{array}$ & $\begin{array}{l}\text { Number of } \\
\text { coefficients } \\
\text { for offset } \\
\text { correction } \\
\text { function }\end{array}$ \\
\hline$\vartheta_{f p a}$ & 174.01 & 227.75 & 13.36 & 16.09 & 4 \\
\hline$\vartheta_{f p a}, \dot{\vartheta}_{f p a}$ & 99.80 & 125.07 & 7.95 & 15.05 & 6 \\
\hline$\vartheta_{f p a}, \vartheta_{T P 1}$ & 46.01 & 111.86 & 8.92 & 14.72 & 6 \\
\hline$\vartheta_{f p a}, \vartheta_{T P 2}$ & 62.82 & 97.73 & 8.03 & 14.72 & 6 \\
\hline$\vartheta_{f p a}, \vartheta_{T P 3}$ & 61.83 & 97.46 & 7.61 & 14.70 & 6 \\
\hline$\vartheta_{T P m}$ & 39.61 & 82.42 & 9.82 & 14.55 & 7 \\
\hline$\vartheta_{f p a}, \vartheta_{T P m}$ & 18.06 & 53.41 & 7.06 & 14.46 & 10 \\
\hline$\vartheta_{f p a}, \dot{\vartheta}_{f p a}, \vartheta_{T P m}$ & 15.24 & 66.68 & 6.99 & 14.61 & 12 \\
\hline$\vartheta_{f p a}, \vartheta_{T P m}, \dot{\vartheta}_{T P m}$ & 14.92 & 73.5 & 6.97 & 15.05 & 13 \\
\hline$\vartheta_{f p a}, \vartheta_{T P m}, \vartheta_{T P m} \cdot \vartheta_{T P n}$ & 17.70 & 56.18 & 6.94 & 14.74 & 14 \\
\hline$\vartheta_{f p a}, \dot{\vartheta}_{f p a}, \vartheta_{T P m}, \dot{\vartheta}_{T P m}$ & 13.70 & 68.74 & 6.91 & 15.36 & 15 \\
\hline$\vartheta_{f p a}, \dot{\vartheta}_{f p a}, \vartheta_{T P m}, \vartheta_{T P m} \cdot \vartheta_{T P n}, \dot{\vartheta}_{T P m}$ & 13.48 & 68.05 & 6.81 & 15.97 & 19 \\
\hline
\end{tabular}

\section{References}

[1] Budzier, H., Gerlach, G., "Thermal Infrared Sensors", John Wiley \& Sons, Chichester (2011).

[2] Tempelhahn, A., Budzier, H., Krause, V., Gerlach, G., "Modeling signal-determining radiation components of microbolometer-based infrared measurement systems", Proc. IRS² 2013, 1-5 (2013); doi: 10.5162/irs2013/iP3.

[3] Budzier, H., "Radiometrische Kalibrierung ungekühlter Infrarot-Kameras", TUDpress, Dresden (2014).
[4] Tempelhahn, A., Budzier, H., Krause, V., Gerlach, G., "Modeling transient thermal behavior of shutter-less microbolometer-based infrared cameras", Proc. SPIE, Vol. 9249, 924904 -1-9 (2014); doi: 10.1117/12.2066624.

[5] ULIS, "Nano 384P TM“, <http://www.ulisir.com/uploads/Products/Nano384P-UL03162.pdf $>, 08 / 2012$.

[6] Nugent, P. W., Shaw, J. A., "Calibration of uncooled LWIR microbolometer imagers to enable long-term field deployment", Proc. SPIE, Vol. 9071, 90710V-1-10 (2014); doi: 10.1117/12.2053082. 